\title{
Une archive audiovisuelle de la création littéraire : les Ateliers d'écriture de Pascale Bouhénic
}

Selina Follonier

\section{(2) OpenEdition}

9 Journals

Édition électronique

URL : https://journals.openedition.org/genesis/4855

DOI : 10.4000/genesis.4855

ISSN : 2268-1590

Éditeur :

Presses universitaires de Paris Sorbonne (PUPS), Société internationale de génétique artistique littéraire et scientifique (SIGALES)

Édition imprimée

Date de publication : 15 décembre 2019

Pagination : 167-176

ISBN : 979-10-231-0650-3

ISSN : $1167-5101$

\section{Référence électronique}

Selina Follonier, « Une archive audiovisuelle de la création littéraire : les Ateliers d'écriture de Pascale Bouhénic », Genesis [En ligne], 49 | 2019, mis en ligne le 01 décembre 2020, consulté le 03 septembre 2021. URL : http://journals.openedition.org/genesis/4855 ; DOI : https://doi.org/10.4000/genesis. 4855 


\title{
Une archive audiovisuelle de la création littéraire : les Ateliers d'écriture de Pascale Bouhénic
}

\author{
Selina Follonier
}

$\mathrm{R}$ approcher la génétique textuelle de l'audiovisuel peut paraître surprenant, si l'on se réfère à la radicale hétérogénéité en termes de matérialité, de circuits de diffusion et de lieux de conservation séparant les sources filmiques des manuscrits littéraires qui forment l'objet d'étude de cette discipline. De même, peu de liens semblent exister du point de vue des outils documentaires et analytiques. En vertu de sa capacité à retracer, conjointement par l'image et par le son, des chaînes d'actions dans le temps, le médium filmique apparaît comme un instrument privilégié pour restituer la «création en acte». Mais son application au domaine de la littérature - contrairement à celui des arts visuels ${ }^{1}$ - se heurte aux apories de l'apparente inadéquation de l'activité scripturale au langage cinématographique. Aussi l'usage critique du vaste corpus de sources audiovisuelles relatives à la vie et à la production littéraires - composé principalement d'interviews, de portraits d'auteurs et d'émissions télévisées -, semble-t-il devoir se restreindre à des approches contextuelles de type biographique, historique ou sociologique, tant il est vrai que ces documents restent muets sur les aspects concrets du travail d'écriture. Les tentatives d'incursions filmiques dans les ateliers d'écrivains dépassent en effet rarement les prises de vues des lieux de travail des auteurs, suivant le rituel de la «visite au grand homme ${ }^{2}$ ». Dans des cas isolés, les réalisateurs procèdent à la captation filmique de brouillons manuscrits, voire à la reconstitution animée de la graphie personnelle d'un auteur, à travers des mises en scène qui obéissent toutefois à des finalités davantage illustratives qu'analytiques ${ }^{3}$. Enfin, l'évocation des enjeux de l'écriture lors d'apparitions des auteurs sur les plateaux de télévision - sans doute la forme la plus fréquente de la rencontre entre littérature et audiovisuel - se limite généralement à l'exégèse de livres publiés (contenu thématique, structure narrative, personnages...), encadrée par des journalistes animés d'une volonté plus ou moins explicite de dévoiler une correspondance entre l'homme et l'œuvre.
À l'aune de ce constat, la série documentaire L'Atelier d'écriture 4 (1994-1998, 2007), réalisée par Pascale Bouhénic et produite par Avidia en partenariat avec le Centre Georges Pompidou, apparaît comme une initiative singulière. Réunissant une douzaine d'entretiens menés avec des auteurs français, la collection vise à cerner «l'écriture derrière l'écrivain, plutôt que l'homme derrière l'œuvres ${ }^{5}$ ». Les propos - exempts de toute anecdote biographique - se focalisent exclusivement sur l'activité d'écriture : modes de conception et de rédaction, techniques de construction des phrases, considérations stylistiques... Les séquences d'entretiens sont complétées par des captations visuelles de manuscrits autographes ou de documents iconographiques associés au travail pré-rédactionnel, ainsi que par des lectures incarnées ou en voix off, accompagnées de projections scripturales. Pareil dispositif, faisant état d'un objectif

(*) Cet article doit beaucoup à Rudolf Mahrer. Qu'il en soit ici vivement remercié.

1. On pense au film consacré par Hans Namuth et Paul Falkenberg à Jackson Pollock (1951), qui a acquis une véritable importance critique en contribuant à la définition du concept d'action painting.

2. À ce sujet, voir Olivier Nora, «La visite au grand écrivain », dans Pierre Nora (dir.), Les Lieux de mémoire, t. II, Paris, Gallimard, 1986, p. 563-587. 3. On se référera par exemple à la tétralogie audiovisuelle que consacre Harald Bergmann au poète allemand Friedrich Hölderlin (Hölderlin Edition, 2013). Le spectateur y voit l'écriture manuscrite de l'auteur taches d'encre comprises - se déposer progressivement sur l'écran.

4. Les entretiens, d'une durée d'une trentaine de minutes chacun, sont menés, dans l'ordre de leur réalisation, avec Olivier Cadiot, Dominique Fourcade, Bernard Heidsieck, Jacques Roubaud, Jude Stéfan, Christian Prigent, Michel Deguy, Valère Novarina, Jean Echenoz, Renaud Camus, Michelle Grangaud et Christian Gailly. Dix ans après la fin du projet, un second film, reconduisant la même formule, est consacré à Olivier Cadiot. Nous remercions chaleureusement Pascale Bouhénic pour les informations qu'elle nous a communiquées au sujet du concept et de la réalisation des Ateliers d'écriture, ainsi que Michel Kaptur (Avidia) pour nous avoir donné accès à la série.

5. Pascale Bouhénic, citée dans Galia Yanoshevsky, L’Entretien littéraire : anatomie d'un genre, Paris, Classiques Garnier, «Études de littérature des XXe et XXI e siècles», 2018, p. 279. 
analytique en partie analogue à celui d'une investigation génétique, invite à s'interroger sur ses potentialités heuristiques dans la perspective de l'élaboration d'un savoir critique sur la genèse des textes. On tentera d'en discuter les enjeux dans les pages qui suivent, tout en articulant cette réflexion à un questionnement plus large sur les relations entre audiovisuel et génétique littéraire.

\section{De l'entretien médiatique comme paratexte génétique}

Envisagés sous l'angle de la typologie des documents génétiques, les entretiens filmés ont, à l'évidence, peu en commun avec les brouillons rédactionnels, plans préparatoires, carnets de notes ou épreuves corrigées qui forment le cœur de ce que l'on définit comme un dossier de genèse. Initialement projetés dans le cadre des Revues parlées du Centre Georges Pompidou, avant d'être édités par cette dernière institution sur support VHS, les films de Pascale Bouhénic peuvent être considérés, selon la terminologie de Gérard Genette, comme des épitextes publics, ayant toutefois ceci de particulier qu'ils ne relèvent pas de la sphère de l'écrit. Leur caractère métadiscursif rapproche les entretiens de ces autres témoins secondaires du processus d'écriture que sont les correspondances, les journaux personnels, les enquêtes journalistiques relatifs au métier d'écrire 6 ou encore les diverses formes d'autocommentaires faisant retour sur la naissance d'œuvres spécifiques, dont Genèse d'un poème (1846) d'Edgar Allan Poe a fondé le genre7. Ils en partagent, respectivement, le principe d'élaboration dialogal, la dimension collective et le mode d'existence public. Du point de vue de leur position temporelle, les entretiens s'inscrivent dans une chronologie différée par rapport au moment de l'écriture; ils sont réalisés le plus souvent (et dans le cas des Ateliers d'écriture exclusivement) après la publication des œuvres auxquelles ils se réfèrent, suivant un intervalle temporel plus ou moins long, pouvant s'étendre du quasi immédiat à plusieurs décennies.

Genre discursif émergé au XIXe siècle dans le contexte de l'essor des cultures médiatiques, l'entretien ${ }^{8}$ est ancré dans la sphère journalistique. Issu de la presse quotidienne, il investit, au fil des mutations technologiques ayant jalonné le siècle suivant, progressivement d'autres supports et espaces médiatiques (radio, télévision...), pour atteindre une extension transmédiale. Sa spécificité générique réside principalement dans la situation de communication qui le sous-tend. L'espace de parole de l'entretien se déploie entre les trois instances que sont l'intervieweur, l'interviewé et le public. Ce dernier, pour être absent des lieux de l'enregistrement, ne constitue pas moins le destinataire principal de l'acte d'interlocution, et revêt une présence virtuelle qui se matérialise à travers l'œil de la caméra et cette «écoute immense et indistincte ${ }^{9} \gg$ que représente le microphone. Si les brouillons manuscrits, de même que les journaux personnels, relèvent de l'espace privé et sont (en principe) destinés à n'être lus que par leur auteur, l'entretien fait l'objet d'une diffusion publique et s'apparente à une performance. Il repose sur des schèmes d'interaction codifiés qui impliquent des enjeux de représentation de soi au sens où ce terme a été défini par Erving Goffman ${ }^{10}$.

Du point de vue du dispositif d'énonciation, l'entretien se fond sur un échange entre (au moins) deux interlocuteurs, ce qui empêche de le considérer comme un genre pleinement auctorial. Comme l'observe Gérard Genette, il constitue

un épitexte [...] doublement médiatisé : par la situation d'interlocution, où les questions, d'une certaine manière, déterminent les réponses, et par l'opération de transmission,

6. Citons, à titre d'exemple, l'enquête «Pourquoi écrivez-vous?», lancée par la revue surréaliste Littérature au tournant de l'année 1920 et reconduite par le journal Libération en 1985, ou encore la série Writers at Work, inaugurée en 1957 par le magazine Paris Review.

7. Voir entre autres les ouvrages de Raymond Roussel, Comment j'ai écrit certains de mes livres (1935), de Thomas Mann, Die Entstehung des Doktor Faustus (1949) et de Hans Magnus Enzensberger, «Wie entsteht ein Gedicht?» (1962), auxquels on peut associer Le Journal des fauxmonnayeurs (1927) d'André Gide.

8. L'entretien littéraire a bénéficié d'un intérêt croissant de la part de la critique au cours de la dernière décennie. Parmi les principales études menées à son sujet, citons l'ouvrage de Galia Yanoshevsky, L'Entretien littéraire : anatomie d'un genre (2018), l'article de David Martens et de Christophe Meurée, «Ceci n'est pas une interview : littérarité conditionnelle de l'entretien d'écrivain » (Poétique, ${ }^{\circ} 177,2015$ ) ainsi que le dossier thématique "L'entretien littéraire» paru dans la revue Argumentation \& Analyse du discours ( $\left.\mathrm{n}^{\circ} 12,2014\right)$, dirigé lui aussi par Galia Yanoshevsky.

9. Philippe Lejeune, Je est un autre : l'autobiographie, de la littérature aux médias, Paris, Le Seuil, «Poétique», 1980, p. 115.

10. Voir Erving Goffman, La Mise en scène de la vie quotidienne, 2 vol., Paris, Minuit, «Le sens commun», 1973. 
qui donne au médiateur, et à l'appareil médiatique dont elle dépend, un rôle parfois très important dans la formulation finale des «propos recueillis » $[\ldots]^{11}$.

Dans les Ateliers d'écriture, l'intervieweur n'apparaît pas sur la bande-image, mais sa présence est attestée par la bande-son, à travers des questions sporadiques qu'il adresse aux écrivains. En tant qu'interlocuteur, il participe pleinement à la construction du discours, se voyant attribuer, selon la visée maïeutique de l'entretien, un rôle de médiateur censé assister l'auteur dans ses réflexions auto-analytiques. Son intervention concerne également le choix et l'agencement des séquences filmiques lors du montage, à travers des opérations de reconfiguration qui peuvent être plus ou moins importantes. L'effet de dilution de l'auctorialité est d'autant plus marqué que les films sont les produits d'un processus d'élaboration collectif qui engage, en plus du réalisateur et des «protagonistes », une multiplicité d'autres acteurs (caméraman, technicien du son, producteur...), ce qui complexifie la question de la responsabilité énonciative du discours.

Sa pluriauctorialité, sa nature journalistique et sa situation anachronique ont de quoi rendre l'entretien suspect dans une perspective génétique. D'autres facteurs potentiellement préjudiciables s'y ajoutent : au même titre que les autocommentaires tardifs auxquels appartient le texte précité de Poe, les propos métadiscursifs s'exposent aux «ambiguïtés de l'auto-analyse ${ }^{12}$ ». Leur caractère rétrospectif pose la question de leur fiabilité, étant donné que les propos s'appuient sur des données mémorielles que l'auteur possède certes de façon privilégiée, voire exclusive, mais qui restent sujettes aux failles et aux imprécisions de la mémoire humaine ${ }^{13}$. Enfin, les discours médiatiques sont réputés pour brouiller les frontières entre réel et fiction; comme le relève Louis Hay, «l'écrivain, dès qu'il s'expose au public, est suspect de retourner à son métier : servir les mots plutôt que les faits 14 ». Cependant, il paraît exagéré de nier aux entretiens toute qualité documentaire et toute pertinence paratextuelle, a fortiori dans le cas d'un dispositif aussi résolument tourné vers les processus d'écriture que celui développé par Pascale Bouhénic. De même, le fait que l'entretien, malgré son statut ambivalent, n'est point absent du paysage des études génétiques - que ce soit en tant que source ou comme méthode d'enquête - autorise à penser que le conditionnement médiatique du discours, s'il appelle une prudence méthodologique correspondante, ne doit pas arrêter toute réflexion 15 .

\section{Récits de genèse : une archive en négatif}

En explorant le concept d'écriture dans la pluralité de ses acceptations, soit au sens à la fois matériel, langagier et artistique du terme, les entretiens de Pascale Bouhénic visent à offrir un aperçu englobant de la fabrique du texte, citations et documents à l'appui. La présentation des «vues d'atelier» se rapproche des formes de médiatisation vidéastiques de dossiers de genèse de bandes dessinées, analysées par Luc Vigier dans un récent numéro de Genesis ${ }^{16}$. La focalisation des échanges s'étend de l'idée originelle des projets rédactionnels jusqu'aux démarches éditoriales, en passant par les différentes étapes de composition, les méthodes de travail et les éventuelles contraintes liées au contexte de production. Les auteurs interviewés se posent en observateurs avertis de leur propre pratique : ils identifient leur démarche d'écriture plus ou moins spontanément comme processuelle ou programmatique, discutent leur usage de différentes catégories de mots ou de signes de ponctuation, abordent des questions relatives aux structures narratives ou aux figures de style, tout en situant leur pratique dans le paysage littéraire de leur temps. Certains n'hésitent pas à livrer des descriptions détaillées de processus endogénétiques, tel Christian Gailly

11. Gérard Genette, Seuils, Paris, Le Seuil, «Poétique», 1987, p. 327. 12. Almuth Grésillon, Éléments de critique génétique : lire les manuscrits modernes, Paris, Presses universitaires de France, 1994, p. 94.

13. Ces mises en garde trouvent une confirmation dans la série de Pascale Bouhénic : Olivier Cadiot, après avoir affirmé s'être détourné de la poésie et rédiger directement en prose, constate, lors d'une confrontation avec les manuscrits de son plus récent roman, la disposition espacée des signes typographiques sur la page, et déclare, étonné mais sans ambages : «donc j'ai menti depuis le début de cet entretien». (Olivier Cadiot dans Pascale Bouhénic, Olivier Cadiot : atelier 2, Paris, Avidia/Centre Pompidou, 2007, 33 : 58.)

14. Louis Hay, La Littérature des écrivains : questions de critique génétique, Paris, José Corti, 2002, p. 287.

15. Nous reprenons cette expression à Gilles Philippe, «Écrire pour parler : quelques problématiques premières », Genesis, n ${ }^{\circ} 39$, «Avantdire», 2014, p. 28.

16. Luc Vigier, «Bande dessinée : une génétique à ciel ouvert», Genesis, $\mathrm{n}^{\circ}$ 43, «Bande dessinée », 2016, p. 51-63. 
qui fait retour sur une technique de composition procédant par fragmentation de phrases d'abord longues, relevant d'une écriture de premier jet, dans le but d'évaluer l'intérêt des différentes composantes et de n'en retenir que l'essentiel. D'autres avancent d'emblée des interprétations économiques des transformations structurelles de leurs textes; ainsi Renaud Camus qui attribue l'abandon d'un dispositif de présentation typographique en deux colonnes à des considérations éditoriales relatives aux coûts d'impression et au prix de vente des livres. L'auteur résume la situation en ces termes : «Il y a des contraintes économiques des formes, savez-vous ça17?»

Quant à évaluer l'utilité génétique des développements métadiscursifs, celle-ci se mesure sans doute à la possibilité de croiser le contenu des entretiens avec des données issues d'autres sources, et notamment avant-textuelles - une possibilité qui dépendra de la nature, du volume et de l'accessibilité des documents manuscrits (physiques ou électroniques) que les auteurs pourront confier un jour à une institution patrimoniale. En l'absence - provisoire ou définitive - de ces documents, toute analyse semble devoir s'en tenir aux réalités matérielles auxquelles les discours peuvent objectivement se rapporter. Si l'on veut hasarder une typologie schématique des indices génétiques que referment les entretiens, elle sera donc nécessairement délimitée, en amont et en aval du processus de rédaction, par le contexte matériel de la production des écrits, les références exogénétiques possédant une existence dans l'espace social et les œuvres publiées.

Dans les limites de la croyance ontologique que l'on peut accorder au médium filmique comme technique d'enregistrement de phénomènes réels, la nature hybride du message audiovisuel permet d'intégrer aux entretiens des reproductions de documents textuels ou iconographiques dont le film fournit une preuve d'existence, voire pérennise, lorsqu'il s'agit de brouillons autographes, un certain état d'(in)achèvement. L'échange avec Olivier Cadiot donne ainsi lieu à des captations visuelles de manuscrits de travail, sous forme de dactylogrammes imprimés et annotés à la main. Dans le portrait dédié à Michelle Grangaud, les images de documents rédactionnels s'associent à celles d'outils d'écriture; il s'agit, entre autres, de captures d'écran d'un logiciel que la poétesse oulipienne utilise pour effectuer les opérations mathématiques présidant à l'élaboration de ses «poèmes timbrés ».
À l'inverse de ces données indicielles, l'ensemble des informations verbales concernant des réalités purement mentales restent, à l'évidence, inexploitables. C'est le cas des multiples témoignages se rapportant à la conception prérédactionnelle de tel poème, recueil ou roman. Les propos de Christian Prigent relatifs à un «schéma formel complètement abstrait, [...] venu d'on ne sait où 18 », que son écriture tente de saisir «plus ou moins efficacement, plus ou moins désespérément ${ }^{19}$ »; ou ceux de Jacques Roubaud concernant sa préférence pour les brouillons mentaux, apportent sans doute un éclairage intéressant sur la complexité des opérations cognitives précédant l'acte d'écrire, mais il serait vain de vouloir y puiser des renseignements substantiels, l'accès à l'intériorité créatrice constituant précisément l'un de ces «renoncements 20 » sur lesquels s'est fondée la génétique textuelle en tant que discipline scientifique.

Situées entre ces deux extrêmes, les indications ayant trait aux modalités et habitudes de travail (environnement, routines, vitesse de rédaction...) ne semblent s'offrir qu'à une exploitation différentielle. Apprendre que Valère Novarina écrit dans des lieux isolés «au cours de séances réglées, très vite ${ }^{21}$ », que Jacques Roubaud compose en marchant ou que Dominique Fourcade s'astreint à des exercices d'écriture quotidiens permet certes d'identifier et de différencier des rythmes individuels, mais ne saurait mettre en lumière les métamorphoses sémantiques et formelles des œuvres en gestation. En revanche, on peut se demander si des mentions relatives à la succession temporelle de différentes étapes de rédaction pourraient fournir des informations utiles en vue de la reconstitution chronologique d'ensembles de pièces avanttextuelles. Une telle utilisation est suggérée par le portrait de Bernard Heidsieck, où l'auteur affirme avoir généralement rédigé par écrit avant de procéder à l'enregistrement sonore de ses poèmes, mais qu'avec le temps, il lui « est arrivé de

17. Renaud Camus dans Pascale Bouhénic, L'Atelier d'écriture de Renaud Camus, Paris, Avidia/Centre Georges Pompidou, 1997, 21 : 15.

18. Christian Prigent dans Pascale Bouhénic, L'Atelier d'écriture de Christian Prigent, Paris, Avidia/Centre Georges Pompidou, 1995, 10:15. 19. Ibid., $10: 25$

20. Daniel Ferrer, «Le matériel et le virtuel : du paradigme indiciaire à la logique des mondes possibles », dans Michel Contat et Daniel Ferrer (dir.), Pourquoi la critique génétique? : méthodes, théories, Paris, CNRS Éditions, 1998, p. 12.

21. Valère Novarina dans Pascale Bouhénic, L'Atelier d'écriture de Valère Novarina, Paris, Avidia/Centre Georges Pompidou, 1996, 38:06. 
composer au magnétophone et sans passer par la page, des textes qu' [il] n'[a] transcrits sur le papier qu'après-coup 22 ». Il semble également possible de reconnaître dans ces propos des indices concernant des modifications des modes de travail tributaires de l'évolution des supports techniques dont disposent les écrivains.

En fin de compte, l'intérêt génétique des entretiens filmés semble se situer principalement sur le versant exogénétique et intertextuel des auto-analyses, en particulier lorsque les éléments concernés sont visuellement représentés et commentés par l'auteur. Si l'évocation, par Olivier Cadiot, de l'intégration de procédés typographiques empruntés à Laurence Sterne dans Futur, ancien, fugitif (1993), ou celle de l'importance de l'œuvre de land art Nid d'eau (2003) de Nils-Udo pour la conception d'Un nid pour quoi faire (2007) n'étonnera pas le lecteur quelque peu familier avec son œuvre et le contexte culturel dans lequel cette dernière s'inscrit, d'autres références, moins évidentes, peuvent être mises au jour au cours des entretiens. Il en est ainsi, par exemple, de la mention de tableaux de William Turner en rapport avec des passages de descriptions figurant eux aussi dans le livre de Cadiot.

Au-delà de la simple identification d'éléments exogénétiques, certains entretiens apportent des précisions sur les circonstances et les modalités d'intégration de ces derniers dans le tissu textuel. Valère Novarina retrace ainsi les recherches qu'il avait menées afin de rassembler les noms de rivières formant l'une des quatre rosaces de La Chair de l'homme (1995). Ils auraient été, d'une part, puisés dans des registres administratifs ou repérés sur des cartes géographiques, d'autre part récoltés au fil de conversations avec des personnes de l'entourage de l'auteur. À moins de ne faire qu'entériner des évidences, l'entretien semble ainsi pouvoir fournir des précisions concernant des sources externes, indiquer des pistes d'investigation, confirmer ou infirmer des hypothèses, mais aussi rendre apparentes les limites de l'archive, notamment lorsqu'il signale l'existence de sources dont il ne subsiste aucune trace matérielle, telles que des formes interactionnelles non écrites. En dernière instance, les discussions relatives aux «influences » ayant marqué les parcours des écrivains, une thématique fortement présente dans les entretiens de Pascale Bouhénic, particularisent l'inscription des auteurs dans un contexte intellectuel et artistique, et mettent en perspective un fonds de connaissances culturelles propre à une époque et à un espace géographique donnés.

\section{D'une poétique des formes à un ethos des processus}

Si les entretiens des Ateliers d'écriture ne sauraient être assimilés à la catégorie des témoins ${ }^{23}$ proprement dits, recouvrant l'étendue des documents matériels rendant manifestes les dynamiques transformationnelles des textes, ils constituent néanmoins des témoins d'une autre réalité, à savoir celle du rapport qu'entretiennent les écrivains à leur pratique littéraire. Pour ne nous renseigner que de manière indirecte sur les opérations linguistiques et scripturales présidant à la fabrication des œuvres, ils nous renseignent de manière directe sur les représentations qui sous-tendent l'activité à laquelle se vouent les auteurs - des représentations qui, en tant qu'elles possèdent une réalité discursive et conceptuelle, sont analysables avec les outils de l'analyse du discours. Elles sont liées à des imaginaires et à des schèmes de figuration qui s'inscrivent dans une dynamique historique et appartiennent, au même titre que les outils d'écriture, au fonds culturel sur lequel se développent les pratiques d'écriture. De ce point de vue, la distance temporelle entre le moment de l'écriture et celui du commentaire, mais aussi le caractère public de la situation d'interlocution, apparaissent comme des facteurs favorisant une porosité accrue des discours aux traditions de représentation collectives 24 .

Il ne s'agira pas ici de questionner la conformité ou la divergence des discours par rapport aux pratiques réelles; que ces dernières ne correspondent pas toujours entièrement aux descriptions qu'en font les intéressés, l'exemple des brouillons raturés d'écriture automatique des surréalistes l'aura montré, mais sans aucunement vouloir imputer aux

22. Bernard Heidsieck dans Pascale Bouhénic, L'Atelier d'écriture de Bernard Heidsieck, Paris, Avidia/Centre Georges Pompidou, 1994, 17: 51. 23. La notion de témoin est définie par Almuth Grésillon comme désignant un «document écrit qui témoigne de la genèse du texte». (Almuth Grésillon, Éléments de critique génétique : lire les manuscrits modernes, op. cit., p. 246. Nous soulignons.)

24. Anthony Glinoer et Vincent Laisney proposent une analyse éclairante des modes d'articulation entre réalités sociales et représentations littéraires dans leur ouvrage consacré aux sociabilités artistiques du XIXe siècle. Voir Anthony Glinoer et Vincent Laisney, L'Âge des cénacles : confraternités littéraires et artistiques au XIXe siècle, Paris, Fayard, 2013. 
auteurs des discours orientés par de seules visées théoriques, il semble pertinent de les examiner sous l'angle de leurs résonances culturelles plus larges.

Pour prendre un exemple parmi les plus manifestes, la question de la quantité du travail pré-rédactionnel et celle de la rapidité d'écriture ont fait l'objet de polarisations majeures selon les époques historiques et les courants esthétiques. La confrontation entre les idéaux prônés par la poésie romantique et le roman naturaliste le met en évidence : tandis que la première véhicule une conception de l'écriture comme jaillissement spontané relevant d'une inspiration quasi extatique qui serait le trait distinctif de l'artiste-génie, le second valorise une démarche de préparation minutieuse, sous forme d'enquêtes documentaires approfondies qui visent à atteindre une fidélité maximale dans la restitution de faits sociohistoriques. Face à ces clivages conceptuels, les écrivains interviewés par Pascale Bouhénic évoquent, avec une notable unanimité, la nécessité d'une dialectique entre un état de réceptivité propice à l'inspiration et un travail régulier, indispensable au perfectionnement stylistique. L'aspect formel est en effet fortement valorisé, et c'est en fonction de cette priorité que les romanciers Jean Echenoz et Christian Gailly affirment renoncer à des enquêtes documentaires pour privilégier le travail de la langue et celui de l'imagination. Parmi les poètes, aussi bien Jude Stéfan que Dominique Fourcade insistent sur l'importance d'un temps de mûrissement nécessaire à la création poétique et à l'approfondissement de leurs recherches formelles. Sans doute faut-il reconnaître dans ces déclarations l'expression d'un discours d'époque, ou du moins de préoccupations esthétiques convergentes au sein d'un groupe d'auteurs dont presque tous sont affiliés aux Éditions de Minuit et P.O.L.

Les distinctions les plus nettes entre les différents témoignages concernent l'évocation de références intermédiales, révélatrices de l'hybridation des pratiques artistiques contemporaines. Tandis que Dominique Fourcade estime avoir tiré des enseignements méthodologiques de la contemplation de tableaux de Matisse, et que Christian Gailly se réclame de techniques de composition utilisées dans la musique jazz, Jean Echenoz fait part de ses aspirations à transposer des procédés cinématographiques dans l'écriture littéraire. Ces affirmations correspondent sans doute à des affinités artistiques personnelles mais sont tout autant des indices traduisant des positionnements au sein du champ culturel.
En effet, il ne paraît pas tout à fait insignifiant qu'un auteur se réfère au parler populaire (Michelle Grangaud) ou à des traités de rhétorique classique (Michel Deguy), ni de voir deux auteurs qualifier un même procédé d'énumération de noms propres respectivement de «ready-made 25 »(Christian Prigent au sujet de «La liste des langues que je parle») et de «sépulture verbale 26 » (Valère Novarina à propos des sobriquets figurant dans La Chair de l'homme).

Les modes de présentation des techniques d'écriture révèlent également le lien inextricable entre description de méthodes et théorisation de ces mêmes méthodes. En effet, à l'écoute des entretiens des Ateliers d'écriture, on ne manque pas de noter combien les propos réflexifs des écrivains oscillent entre le comment, le pourquoi et la supposée signification de l'acte d'écrire. Les discours se fondent sur un enchevêtrement complexe de déclarations ayant tous trait au travail rédactionnel, mais qui, en réalité, mêlent aux récits de genèse proprement dits des prises de position théoriques et des professions de foi esthétiques, chacun livrant non seulement son témoignage sur sa manière d'écrire, mais aussi sa vision de l'histoire littéraire, son art poétique et sa conception de la littérature.

Cet enchevêtrement devient particulièrement manifeste lorsque les entretiens abordent la question de l'intentionnalité. En thématisant la plus ou moins grande maîtrise qu'ils possèdent sur le devenir des textes et la plus ou moins grande conscience des modes d'engendrement des œuvres, les écrivains ne prennent pas seulement part à un débat qui divise la critique 27 , mais font fréquemment appel à des concepts qui se situent hors de la sphère de l'esthétique. Interrogé sur la manière dont naissent ses écrits, Valère Novarina évoque l'état de dépossession dont il fait l'expérience lorsqu'il rédige : «Ce qui se passe avec les mots, je ne sais pas trop. Ils bougent tout le temps ${ }^{28}$. $\gg$ L'auteur rejoint sur ce point

25. Christian Prigent dans Pascale Bouhénic, L'Atelier d'écriture de Christian Prigent, op. cit., 08:45.

26. Valère Novarina dans Pascale Bouhénic, L'Atelier d'écriture de Valère Novarina, op. cit., 18:44.

27. Au sujet de l'intentionnalité, voir la synthèse de Rudolf Mahrer, «Une idée derrière la tête ? : intention et production écrite», dans PierreMarc de Biasi et Anne Herschberg Pierrot (dir.), L'Euvre comme processus, Paris, CNRS Éditions, 2017, p. 207-219.

28. Valère Novarina dans Pascale Bouhénic, L'Atelier d'écriture de Valère Novarina, op. cit., 15:24. 
Dominique Fourcade qui explique avoir été entraîné, lors de la rédaction de Rose-déclic (1984), par une «mécanique extrêmement puissante 29 » et n'avoir pu s'en libérer qu'avec grande difficulté, une fois le livre terminé. Toutefois, chez Novarina, le foisonnement des mots et les «affinités mys-

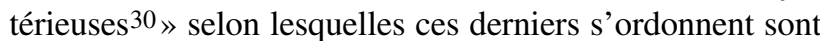
reliés à un imaginaire religieux (l' «énergie fondamentale de la langue qu'est le Verbe $\left.{ }^{31} \gg\right)$, ce qui modifie considérablement la teneur des propos. De telles associations entre processus scripturaux, expériences d'écriture et principes éthiques peuvent être mobilisées de façon très explicite : Michelle Grangaud s'y emploie quand elle décrit le principe d'impersonnalité inhérent aux méthodes de composition oulipiennes au moyen d'explications puisant dans le champ sémantique de la démocratie ${ }^{32}$. En joignant la plus grande abstraction formelle à un idéal social, elle établit une équivalence entre une technique d'écriture et un idéal politique - un idéal dont on n'est pas étonné qu'il ne se retrouve pas chez son confrère Jacques Roubaud, lui aussi Oulipien notoire, qui présente ces mêmes procédés sous l'angle de son admiration pour la beauté des axiomes mathématiques.

Les descriptions des méthodes de travail n'échappent donc pas aux enjeux symboliques de ce «chantier de construction de [1']ethos [auctorial] ${ }^{33} \gg$ qu'est l'entretien littéraire. En se prononçant non seulement sur les choix et les implications de telle ou telle démarche, mais en conférant à ces choix une portée quasi philosophique, les récits de genèse deviennent des stratégies paratextuelles dotées d'une force pragmatique et se voient mises au service de l'affirmation de postures poétiques spécifiques. Ces observations rejoignent les constats que tire Galia Yanoshevsky dans un récent ouvrage 34 consacré à l'entretien littéraire : selon l'auteur, ce genre discursif gagne à être envisagé comme une forme de création, situé dans un espace générique distinct mais non complètement détaché de la sphère de l'œuvre littéraire dont il forme un prolongement.

Sous l'enquête mise en place par les Ateliers d'écriture, on voit se profiler un véritable imaginaire des processus d'écriture, opérant à la jonction entre «mythologies intimes et [...] légendes collectives ${ }^{35} »$. Cet imaginaire est lié mais non coextensif à celui de la figure de l'écrivain ${ }^{36}$, dont les déclinaisons se trouvent visuellement actualisées dans les entretiens filmés (accessoires comme la cigarette, focalisation de la caméra sur des objets symboliques que sont les tables de travail et les bibliothèques personnelles...). Les récits de genèse puisent dans et contribuent à alimenter un réservoir de métadiscours qui circulent dans l'espace social au moyen de représentations discursives et médiatiques, dont les écrivains sont à la fois les héritiers et les relais. On notera à ce propos que Valère Novarina se réfère, lorsqu'il présente l'acte d'écrire comme nécessitant l'implication du corps entier du scripteur, à des pratiques de «somatisation ${ }^{37}$ » employées par des écrivains du XVII ${ }^{\mathrm{e}}$ siècle, tels que Bossuet qui, pour écrire, «s'entourait de bandelettes » tandis que tel autre «se mettait les pieds dans la glace 38 ». À travers de telles comparaisons, les discours auto-poiétiques acquièrent un pouvoir performatif, permettant à l'auteur de s'inscrire dans une généalogie littéraire ${ }^{39}$.

29. Dominique Fourcade dans Pascale Bouhénic, L'Atelier d'écriture de Dominique Fourcade, Paris, Avidia/Centre Georges Pompidou, 1994, $17: 17$.

30. Ibid., 21:34.

31. Ibid., 16:05.

32. La poétesse affirme entre autres que «pour [elle], fondamentalement, les lettres sont égales », et file la métaphore de l'équivalence entre hommes et signes de l'alphabet («Chacun de nous est une lettre»). (Michelle Grangaud dans Pascale Bouhénic, L'Atelier d'écriture de Michelle Grangaud, Paris, Avidia/Centre Georges Pompidou, 1998, 04:00 et 06:10). 33. Galia Yanoshevsky, «L'entretien littéraire - un objet privilégié pour l'analyse du discours?», Argumentation \& Analyse du discours, $\mathrm{n}^{\circ} 12$, «L'entretien littéraire», 2014, journals.openedition.org/aad/1726 [consulté le 5 juillet 2019].

34. L'ouvrage précité de Galia Yanoshevsky explore cette hypothèse d'une dimension créatrice et esthétique de l'entretien littéraire. Voir Galia Yanoshevsky, L'Entretien littéraire : anatomie d'un genre, op. cit.

35. Sylvie Ducas, «Quand l'entretien littéraire se fait enquête sociologique : discours de la reconnaissance littéraire et posture ambivalente de 1'écrivain consacré », Argumentation \& Analyse du discours, $\mathrm{n}^{\circ} 12$, «L'entretien littéraire », 2014, journals.openedition.org/aad/1698 [consulté le 5 juillet 2019].

36. Au sujet des figurations de l'écrivain, voir en particulier José-Luis Diaz, L'Écrivain imaginaire : scénographies auctoriales à l'époque romantique (2007) et Jérôme Meizoz, La Littérature "en personne» : scène médiatique et formes d'incarnation (2016), ainsi que l'ouvrage collectif dirigé par David Martens et Myriam Watthée-Delmotte, L'Écrivain, un objet culturel (2012).

37. Valère Novarina dans Pascale Bouhénic, L'Atelier d'écriture de Valère Novarina, op. cit., 36:57.

38. Ibid., 37:07.

39. En ce qui concerne l'évocation des généalogies artistiques dans les entretiens littéraires, voir Sylvie Ducas, «Quand l'entretien littéraire se fait enquête sociologique : discours de la reconnaissance littéraire et posture ambivalente de l'écrivain consacré», art. cit. 
Rappelons enfin que la teneur conceptuelle des représentations de l'acte d'écrire peut non seulement orienter les pratiques mais également avoir des conséquences matérielles. En effet, les propos métadiscursifs cristallisent des logiques et des valeurs qui correspondent à celles œuvrant dans un autre domaine primordial pour la génétique textuelle : celui des archives. Au même titre que la publication d'un livre ou l'autoprésentation lors d'un entretien, le dépôt d'un fonds d'archives participe, pour les auteurs, de cette entreprise de réécriture de soi qui caractérise la construction d'une identité publique. Il ne reste plus à démontrer combien le geste de confier des ensembles documentaires à des institutions patrimoniales fait l'objet de stratégies de sélection et d'arbitrages de la part de leurs propriétaires, soucieux de maitriser non seulement l'image médiatique qu'ils construisent de leur vivant, mais aussi l'image posthume inscrite dans ce corpus que représentent les traces matérielles de leur activité. C'est en effet sur cette «face cachée 40 » de la génétique (celle de ses conditions de possibilité) que sont la conservation et la transmission des documents d'archives, que se négocie ce qui, en dernière instance, n'apparaît que comme un autre mode de (pré)construction d'un récit de genèse...

\section{Un imaginaire médiatique de la création littéraire}

Le statut épistémologique des entretiens filmés des Ateliers d'écriture s'avère complexe dans la mesure où il renvoie à des champs théoriques hétérogènes et à l'ambivalence de la place de l'audiovisuel dans le domaine culturel et scientifique. Leur valeur heuristique se fonde sur deux modes d'appréhension en apparence contradictoires, à savoir, d'une part, celui de document, et d'autre part celui d' 'euvre (au sens de création discursive et filmique). À condition de ne pas reculer devant ce paradoxe, mais de considérer les deux facettes dans leur complémentarité, on y retrouvera la dualité constitutive de tout acte d'écriture, production à la fois matérielle et intellectuelle.

Au-delà de la mise en évidence d'éventuels indices contextuels, endogénétiques ou intertextuels, les entretiens filmés éclairent la façon spécifique dont chacun des écrivains se positionne face à son travail, ainsi que les manières contemporaines de rendre compte des processus créatifs (dans les limites, cela s'entend, de l'échantillon des personnalités ayant pris part au projet). On ne peut que deviner l'intérêt que représenteront ces sources dans cinquante ou cent ans - non seulement pour de futures études génétiques des œuvres des auteurs interviewés, mais aussi parce qu'elles révéleront alors pleinement leur historicité41. En mettant en lumière les liens qui se tissent entre pratiques d'écriture et représentations symboliques, ainsi que la manière spécifique dont celles-ci s'articulent à l'histoire littéraire et aux courants esthétiques d'une époque, les Ateliers participent à l'historicisation du concept d'écriture et contribuent ainsi à une histoire culturelle de l'écriture.

On mesure l'intérêt que représenterait une étude comparative, diachronique et transmédiale, voire transnationale, de tels entretiens, autocommentaires ou récits de genèse. Le projet de Pascale Bouhénic s'inscrit, nous l'avons vu, dans une lignée de productions similaires, parmi lesquels il convient de citer les Werkstattgespräche mit Schriftstellern (1962) réunis par Horst Bienek, les Théâtres d'écritures (1993) présentés par Yves Bridel et Adrien Pasquali, ainsi que l'emblématique collection Les Sentiers de la création (1969-1976) publiée par l'éditeur Albert Skira. À une trentaine d'années de distance, les Ateliers d'écriture poursuivent le programme tracé par cette dernière, en se proposant pareillement de réunir des réflexions d'écrivains sur l'acte d'écrire et en associant aux métadiscours des reproductions de manuscrits ou de documents iconographiques. Mobilisant, comme leur ancêtre, le dispositif de la collection - opérateur de patrimonialisation par excellence -, ils visent à constituer une véritable archive audiovisuelle de la création littéraire, à une époque où la patrimonialisation du littéraire passe progressivement par d'autres voies que celles de l'imprimé. Le terme archive doit dans ce contexte être compris sous l'angle de son évolution sémantique contemporaine, marquée par ce que Jean-François Bert identifie comme un mouvement

40. Louis Hay, La Littérature des écrivains : questions de critique génétique, op. cit., p. 308.

41. Production relativement confidentielle, diffusée principalement dans les milieux littéraires et dans des écoles d'art, la collection Ateliers d'écriture fait son retour sur les devants de la scène grâce à des projections de certains épisodes lors de colloques récents, consacrés respectivement à Michel Deguy (Cerisy, 2006) et à Olivier Cadiot (Université Paris Diderot, 2015). En même temps, elle entre dans les bibliographies critiques des auteurs et, sous forme de transcriptions, dans des livres d'entretiens (voir infra, note 45). 
d'«extension du domaine des archives 42 ». En effet, suite à l'essor des nouvelles techniques d'enregistrement et de diffusion, et dans le sillage d'entreprises comme celles des Archives de la parole de Ferdinand Brunot ou des Archives de la planète d'Albert Kahn, le terme ne se restreint plus aux lieux de conservation institutionnels réunissant des fonds documentaires, mais s'étend à des formes de collecte active et de stockage de données.

Les médias audiovisuels s'emploient ainsi, en marge des circuits traditionnels de conservation et de transmission des savoirs, à bâtir une mémoire médiatique à laquelle les écrivains participent en proportion des nouveaux impératifs d' «audiovisibilité» liés à leur métier. S'il est vrai que les gisements d'informations paratextuelles à intérêt génétique demeurent rares et difficiles à repérer dans les méandres des archives audiovisuelles, ils ne sont pas inexistants pour autant. Un autre bel exemple d'une association entre réflexion génétique et patrimonialisation audiovisuelle est donné par l'entretien d'Alain Robbe-Grillet, conduit par Benoît Peeters pour l'IMEC et publié en 2001 sur DVD. Cet entretien-fleuve de 375 minutes dresse une rétrospective dialoguée de la vie et de l'œuvre de l'auteur des Gommes, tout en y associant des projections de manuscrits accompagnées de commentaires. À part ces dispositifs exclusivement conversationnels, d'autres initiatives se tournent vers le genre du reportage. Il en est ainsi du film Pierre Guyotat : 52 minutes dans la langue (1989), réalisé par Ludwig Trovato. Conçu comme une exploration conjointe de l'univers et du mode de travail de Pierre Guyotat, ce documentaire inclut des scènes montrant l'auteur en train de dicter un texte à un collaborateur ${ }^{43}$. Loin de se borner à rassembler des propos métadiscursifs, la «visite d'atelier» peut donc également aboutir à la saisie d'œuvres encore en gestation. Un portrait télévisé consacré à Nicolas Bouvier dans le cadre de l'émission Trois jours avec... (1975) donne lieu à la captation filmique de documents préparatoires d'un roman qui s'intitulait alors La Zone de silence et n'a paru que six ans plus tard sous le titre Le Poisson-scorpion (1981), aboutissant ainsi à une diffusion massive d'avanttextes par la voie des chaînes hertziennes ${ }^{44}$. Le format de cette série de reportages se rapproche de celui de l'émission Euvres en chantier, produite par la Radio-Télévision Belge Francophone (RTBF), qui donne la parole aussi bien à des écrivains qu'à des artistes, des compositeurs et des intellectuels. Si les épisodes ne correspondent pas tous au programme que suggère le titre, la série n'en comporte pas moins des enquêtes approfondies sur des formes de création littéraire ou artistique, comme par exemple la danse dans le cas d'un portrait dédié à Michèle Noiret, une chorégraphe que l'équipe de télévision accompagne lors de la préparation de son spectacle Twelve Seasons (2001).

Reste que de telles sources demeurent le plus souvent invisibles aux yeux des études littéraires. Non seulement en raison de résistances disciplinaires face à des objets qui relèvent de modes de productions en partie déterminés par des logiques commerciales et dotés d'une faible légitimité culturelle, mais également du fait de l'éclatement des lieux de conservation. À moins de figurer sous forme de copies physiques dans les archives personnelles des écrivains (ou d'entrer, grâce à des transcriptions, dans des livres d'entretiens, où ils accèdent à un mode d'existence plus institutionnalisé ${ }^{45}$ ), ces productions restent confinées dans des archives audiovisuelles qui sont certes de plus en plus faciles d'accès grâce à de vastes campagnes de numérisation, mais qui se trouvent dans une situation de non-contiguïté par rapport aux archives littéraires qui constituent le terrain d'investigation ordinaire des chercheurs en lettres 46 . Enfin, leur statut fonctionnel ambigu - à la fois dispositif analytique,

42. Jean-François Bert, «Pratiques d'archives : problèmes actuels sur les usages du matériau documentaire », dans Véronique Ginouvès et Isabelle Gras (dir.), La Diffusion numérique des données en SHS : guide des bonnes pratiques éthiques et juridiques, Aix-en-Provence, Presses universitaires de Provence, 2018, p. 31-38.

43. Si l'intérêt de telles images est indéniable, il faut toutefois rappeler que même les productions filmiques obéissant aux codes génériques du documentaire et aspirant à un idéal d'objectivité comportent ceci d'ambigu que la présence de la caméra altère la réalité qu'elle enregistre.

44. À ce sujet, voir Selina Follonier, «Trois jours avec..., ou la littérature au quotidien : écrivains et écriture au prisme de l'entretien-reportage télévisé (1973-1976) », A contrario, n² 27, 2018, p. 117-135.

45. C'est le cas du livre d'entretiens Improvisations et arrangements (2018) de Dominique Fourcade, contenant une transcription partielle de l'interview menée par Pascale Bouhénic.

46. Il convient de mentionner ici deux initiatives institutionnelles s'employant à établir un pont entre archives littéraires et archives audiovisuelles : d'une part le Centre d'archives Gaston-Miron, rattaché à l'Université de Montréal, qui réunit et met à la disposition des chercheurs des sources audiovisuelles relatives à la vie littéraire québécoise ; d'autre part le projet IMVOCS (Images et voix de la culture suisse), destiné à la collecte et à la sauvegarde d'enregistrements sonores et filmiques d'écrivains dont les fonds sont déposés aux Archives littéraires suisses (ALS). 
vecteur de médiation culturelle et œuvre filmique - ainsi que l'absence de normes et de principes méthodologiques explicites encadrant la pratique documentaire ${ }^{47}$ semblent les priver de la possibilité de se voir accorder un crédit scientifique analogue à celui d'études historiques écrites dont elles partagent pourtant souvent les visées.

La question de la légitimité épistémologique des sources filmiques gagne cependant en actualité dans le champ de la génétique. En témoigne l'intégration de l'audiovisuel dans les activités de recherche de l'ITEM, à travers la réalisation de plusieurs documentaires au sein de l'équipe "Génétique des arts visuels», effectuée sous l'impulsion de Monique Sicard. Entre 2012 et 2016, trois films ont été consacrés respectivement aux photographes Jean-Marie Fadier, Hsieh Chun-Te et Jean-Michel Fauquet. Véritables enquêtes associant entretiens avec les artistes, visites de lieux ayant servi de décor à des créations photographiques, voire suivi en temps réel de l'exposition de papiers photosensibles, ils emploient la caméra comme un instrument d'investigation scientifique. En tant qu'outil d'analyse, celle-ci permet entre autres, comme le souligne Monique Sicard, de «rendre manifeste le temps long de la création photographique ${ }^{48}$ ». On peut se demander si de telles initiatives se prolongeront dans l'avenir - y compris dans des domaines autres que celui des arts visuels. Sans doute serait-ce l'occasion d'envisager de manière renouvelée la question de l'opérativité scientifique de l'audiovisuel et de procéder à une évaluation théorique de sa position, ainsi que de celle de l'entretien, au sein des études génétiques.

47. On pense, entre autres, à l'usage de la musique dont l'impact sur les modes de réception du message audiovisuel s'avère décisif. (Sur cette question, voir Michel Chion, L'Audio-vision : son et image au cinéma, Paris, A. Colin, 1990.)

48. Monique Sicard, entretien non publié, propos recueillis par Selina Follonier, juillet 2019. Nous remercions Monique Sicard pour les informations qu'elle nous a transmises au sujet de ces documentaires.

SELINA FOLLONIER est assistante-doctorante au Centre des sciences historiques de la culture de l'Université de Lausanne. Elle prépare une thèse intitulée Vers une histoire audiovisuelle de la littérature : enjeux, dispositifs, approches théoriques et est l'auteur de plusieurs articles portant sur les rapports entre littérature et audiovisuel. Ses domaines de recherche concernent l'historicisation de la littérature par les médias, le patrimoine audiovisuel de la littérature et les archives audiovisuelles du littéraire. 


\section{Une archive audiovisuelle de la création littéraire : les Ateliers d'écriture de Pascale Bouhénic}

$\mathbf{S}_{i}$ les entretiens d'écrivains semblent, le plus souvent, n'offrir à l'investigation critique que des informations contextuelles de type biographique, historique ou sociologique, les entretiens filmés de la série L'Atelier d'écriture, focalisés exclusivement sur les processus d'écriture, dérogent à la règle. Faisant état d'une visée analytique en partie analogue à celle d'une enquête génétique, ils invitent à s'interroger sur leurs potentialités heuristiques dans la perspective de l'élaboration d'un savoir critique sur la genèse des textes. Comment envisager, d'un point de vue théorique, les informations qu'ils contiennent, compte tenu de la complexité du dispositif énonciatif qui les sous-tend et de l'ambivalence de leur statut, entre métadiscours et œuvre autonome ? Comment se rapportent-ils à d'autres formes de documentation du travail d'écriture? Comment concevoir l'articulation des fonctions documentaire, patrimoniale et esthétique dont ils sont investis? Cet article tentera d'élucider ces enjeux, tout en ouvrant à un questionnement plus large sur les relations entre audiovisuel et génétique littéraire.

If writers' interviews seem, most of the time, to offer to the critical investigation only contextual information (biographical, historical, sociological...), the filmed interviews of the L'Atelier d'écriture series, focused exclusively on the writing processes, derogate from the rule. Presenting an analytical aim partly analogous to that of a genetic investigation, these sources invite to question their heuristic potential in the perspective of the elaboration of a critical knowledge on the genesis of texts. How can one envisage, from a theoretical point of view, the information that they contain, given the complexity of the enunciative device behind them and the ambivalence of their status, between metadiscourse and autonomous work of art? How do they relate to other forms of documentation concerning the writing process? How can one conceive the articulation of the documentary, the patrimonial and the aesthetic function with which they are invested? This article attempts to elucidate these issues, while opening to a broader questioning on the relations between audiovisual and literary genetics.

Da Autoreninterviews einer kritischen Untersuchung häufig nur kontextbezogene Informationen (biografisch, historisch, soziologisch) zu bieten scheinen, konzentrierten sich die filmischen Interviews der Reihe L'Atelier d'écriture ausschließlich auf die Schreibprozesse und weichen damit von der Regel ab. Mit analytischem Blick, der teilweise einer genetischen Untersuchung entspricht, laden die Interviews dazu ein, deren heuristischen Potentiale hinsichtlich der Entwicklung von kritischen Kenntnissen über die Textgenese zu untersuchen. Wie kann man sich die Informationen, die sie enthalten, in theoretischer Hinsicht vorstellen, auch unter Berïcksichtigung der Komplexität des ihnen zugrunde liegenden Ausdrucksmittels und der Ambivalenz ihres Status zwischen Metadiskurs und autonomen Kunstwerk? In welcher Beziehung stehen sie zu anderen Dokumentationsformen von Schreibtätigkeit? Wie äußern sich die dokumentarischen, patrimonialen und ästhetischen Funktionen, die in ihnen angelegt sind? Dieser Artikel versucht diese Themen zu erläutern, um gleichzeitig eine breitere Fragestellung der Verhältnisse zwischen audiovisueller und literarischer génétique zu ermöglichen.

A menudo las entrevistas de escritores parecen no poder ofrecer a la investigación crítica nada más que informaciones contextuales de tipo biográfico, histórico o sociológico; las entrevistas filmadas de la serie L'Atelier d'écriture, centradas exclusivamente en los procesos de escritura, constituyen una excepción a la regla: al perseguir una finalidad analítica análoga, en parte, a la de un estudio genético, suscitan un interrogante acerca de sus potencialidades heurísticas en la perspectiva de la elaboración de un discurso crítico sobre la génesis de los textos. ¿Cómo considerar, desde un punto de vista teórico, las informaciones que contienen, teniendo en cuenta la complejidad del dispositivo enunciativo que las estructura y la ambivalencia de su condición, entre metadiscurso y obra autónoma? ¿Cómo se relacionan con otras formas de documentación del trabajo de escritura? ¿Cómo concebir la articulación de las funciones documental, patrimonial y estética que las constituyen? Este artículo intentará dilucidar esos temas, abriendo un acceso a una interrogación más amplia sobre las relaciones entre audiovisual y genética literaria.

S e as entrevistas dos escritores parecem, na maior parte das vezes, oferecer à investigação crítica, apenas informações contextuais do tipo biográfico, histórico ou sociológico, as entrevistas filmadas da série L'Atelier d'écriture, focadas exclusivamente nos processos de escrita, fogem à regra. Dando conta de uma visão analítica, em parte análoga a uma enquete genética, essas entrevistas convidam a se interrogar sobre suas potencialidades heurísticas na perspectiva da elaboração de um saber crítico sobre a gênese dos textos. Como considerar, de um ponto de vista teórico, as informações que elas contém, tendo em conta a complexidade do dispositivo enunciativo implícito e a ambivalência de seu estatuto, entre meta-discurso e obra autônoma ? Como elas se referem à outras formas de documentação do trabalho de escrita ? Como conceber a articulação das funções documentária, patrimonial e estética nas quais elas estão inseridas ? Este artigo tentará elucidar estas questões de maneira a abrir um questionamento mais amplo sobre as relações entre audiovisual e genética literária.

Se le interviste agli scrittori, il più delle volte, sembrano offrire alla critica nient' altro che una serie d'informazioni biografiche, storiche o sociologiche, le interviste filmate della serie L'Atelier d'écriture, centrate unicamente sul processo di scrittura, rappresentano un'eccezione a questa regola. Manifestando un'ambizione analitica, in parte analoga a quella di un'inchiesta genetica, esse invitano a interrogarsi sulle loro potenzialità euristiche nella prospettiva dell'elaborazione di un sapere critico sulla genesi dei testi. Come valutare, da un punto di vista teorico, le informazioni che contengono, tenuto conto della complessità del dispositivo d'enunciazione che le sottende e dell' ambivalenza del loro statuto, tra meta-discorso e opera autonoma? Come concepire l'articolazione delle funzioni documentale, patrimoniale ed estetica di cui sono portatrici? L'articolo proverà a delucidare questi temi, aprendo a una problematica più ampia sulle relazioni tra audiovisivo e genetica letteraria. 